\title{
The Regulation of Bacterial Biofilm Formation by CAMP-CRP: A Mini-Review
}

\author{
Cong Liu, Di Sun, Jingrong Zhu, Jiawen Liu and Weijie Liu*
}

School of Life Sciences, Jiangsu Normal University, Xuzhou, China

Biofilms are communities of microorganisms that live in a self-produced extracellular matrix in order to survive in hostile environments. Second messengers, such as c-diGMP and cAMP, participate in the regulation of biofilm formation. c-di-GMP is a major molecule that is involved in modulating the bacterial transition between a planktonic lifestyle and biofilm formation. Aside from regulating carbon catabolism repression in most bacteria, CAMP has also been found to mediate biofilm formation in many bacteria. Although the underlying mechanisms of biofilm formation mediated by CAMP-CRP have been well-investigated in several bacteria, the regulatory pathways of CAMP-CRP are still poorly understood compared to those of c-di-GMP. Moreover, some bacteria appear to

OPEN ACCESS

Edited by:

Christian Sohlenkamp,

National Autonomous University

of Mexico, Mexico

Reviewed by:

Serena Rinaldo,

Sapienza University of Rome, Italy

Wenli Chen,

Huazhong Agricultural University,

China

${ }^{*}$ Correspondence:

Weijie Liu

leonliu2013@126.com

Specialty section:

This article was submitted to Microbial Physiology and Metabolism,

a section of the journal

Frontiers in Microbiology

Received: 04 February 2020

Accepted: 03 April 2020

Published: 14 May 2020

Citation:

Liu C, Sun D, Zhu J, Liu J and Liu W (2020) The Regulation of Bacterial Biofilm Formation by

CAMP-CRP: A Mini-Review.

Front. Microbiol. 11:802.

doi: 10.3389/fmicb.2020.00802 form biofilm in response to changes in carbon source type or concentration. However, the relationship between the carbon metabolisms and biofilm formation remains unclear. This mini-review provides an overview of the cAMP-CRP-regulated pathways involved in biofilm formation in some bacteria. This information will benefit future investigations of the underlying mechanisms that connect between biofilm formation with nutrient metabolism, as well as the cross-regulation between multiple second messengers.

Keywords: biofilm, second messengers, cAMP-CRP, c-di-GMP, carbon catabolite repression

\section{INTRODUCTION}

Biofilms are structured communities of microorganisms in which cells are embedded in an extracellular matrix (Flemming and Wuertz, 2019). Biofilms are generally formed to promote bacterial survival in harsh environments (Flemming et al., 2016). While biofilm formation is a complex process regulated by several different factors in various bacteria, some regulators of biofilm formation, such as second messengers, are common to nearly all bacteria (Kalia et al., 2013; Jenal et al., 2017). The second messenger c-di-GMP is universally recognized as a "switch molecule" that controls the bacterial transition between a planktonic lifestyle and biofilm formation (Dahlstrom and O'Toole, 2017). High c-di-GMP levels induce biofilm formation, whereas low c-di-GMP levels induce planktonic growth (Valentini and Filloux, 2016; Liu et al., 2017). Another well-characterized second messenger cAMP, the cofactor of CRP, is primarily involved in carbon catabolite repression in bacteria. In Escherichia coli and Salmonella enterica serovar Typhimurium, the uptake and concomitant phosphorylation of preferential carbon sources, such as glucose, requires the phosphoenolpyruvate: sugar phosphotransferase system (PTS) (Deutscher et al., 2006). When PTS sugars are in the medium, the phosphoryl group of phosphorylated EIIA protein (EIIA P) is transferred to PTS sugars in order to complete the uptake of these sugars into the cell (Hogema et al., 1997; Park et al., 2006). When PTS sugars are limited and the non-PTS sugars 
are present in the medium, adenylyl cyclase CyaA is activated directly by EIIA $\sim \mathrm{P}$ to synthetize cAMP (Hogema et al., 1997; Deutscher et al., 2006; Park et al., 2006). Subsequently, cAMPCRP activates the transcription of genes encoding these proteins for the transport of non-PTS sugars, which results in the transport of these non-PTS sugars into the cell (Park et al., 2006). Thus, carbon catabolite repression is achieved by cAMPCRP (Deutscher et al., 2006). In addition, cAMP-CRP is an archetypical global regulator. For instance, this complex regulates the transcription of $7 \%$ of the genes in $E$. coli, which illustrates that the biological role of cAMP goes far beyond carbon catabolite repression and includes other cellular processes such as biofilm formation (Hufnagel et al., 2016).

It is widely accepted that second messengers play a central role in biofilm formation, and there are several reviews regarding the c-di-GMP-mediated regulation of biofilm formation ( $\mathrm{Ha}$ and O'Toole, 2015; Purcell et al., 2016; Valentini and Filloux, 2016; Jenal et al., 2017). The underlying mechanisms of biofilm formation mediated by CAMP-CRP, however, also deserve to be reviewed. First, high cAMP levels influence biofilm formation differently among bacterial species. For example, cAMP-CRP promotes biofilm formation in $E$. coli and Pseudomonas aeruginosa (Müller et al., 2009; Sutrina et al., 2015), but inhibits biofilm formation in Serratia marcescens and Vibrio cholerae (Kalivoda et al., 2013). Moreover, cAMP-CRP is involved in diverse physiological functions, thus connecting physiological processes with biofilm formation (Sutrina et al., 2015, 2019). In this mini-review, we provide an overview of the ancillary role of cAMP-CRP-mediated biofilm formation in different bacteria. This information will benefit future investigations regarding the underlying mechanisms that connect between biofilm formation and other important physiological processes in bacteria.

\section{CAMP-CRP REGULATES BIOFILM FORMATION IN E. cOIi}

Biofilm formation in E. coli is mediated by several carbon sources, including PTS and non-PTS sugars (Sutrina et al., 2015, 2019). Both sugars exert inhibitory effects on biofilm growth at high concentrations, which are reversed by the addition of exogenous cAMP (Sutrina et al., 2015, 2019). It has also been shown that cAMP-CRP, in response to carbon sources, plays an ancillary role in the regulation of biofilm formation in E. coli (Figure 1).

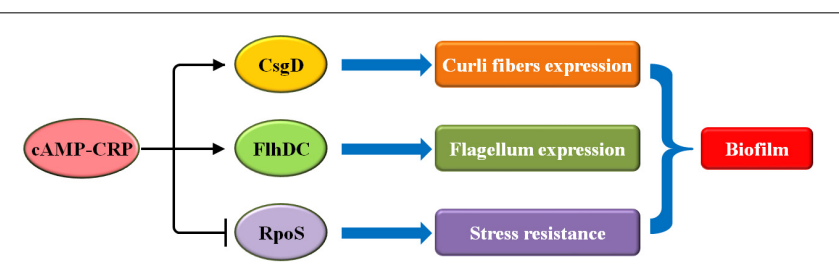

FIGURE 1 | In E. coli, CAMP-CRP regulates biofilm formation though three pathways: CsgD, FlhDC, and RpoS. Arrowheads indicate activation, with the rear of the arrow representing inhibition.
First, cAMP-CRP mediates curli fiber synthesis. Curli fibers, as the part of the extracellular matrix, are involved in the adhesion and attachment of bacteria to surfaces in order to initiate biofilm formation (Barnhart and Chapman, 2006). The synthesis of curli fibers is controlled, at least in part, by six proteins that are encoded by the $\operatorname{csg} B A$ and $\operatorname{csg} D E F G$ operons (Rudd, 2000; Barnhart and Chapman, 2006). CsgD is a major regulator that mediates the expression of both operons (Brombacher et al., 2003; Ogasawara et al., 2011). cAMP-CRP directly activates the transcription of $\operatorname{csg} D$, thereby controlling biofilm formation (Hufnagel et al., 2016).

Second, cAMP-CRP regulates flagellum biosynthesis. The flagellum is a critical organelle involved in bacterial motility and chemotaxis (Xie et al., 2011; Guttenplan and Kearns, 2013). During biofilm formation in E. coli, the flagella act as a mechanosensory, sensing the solid surface signals and driving the initial attachment (Belas, 2014). Flagellum biosynthesis in E. coli involves approximately 50 gene clusters that are controlled by the master motility complex FlhDC (Chevance and Hughes, 2008; Guttenplan and Kearns, 2013). cAMP-CRP positively regulates the transcription of $f h D C$, thus mediating flagellumassociated physiological processes, including biofilm formation (Soutourina et al., 1999).

Third, cAMP-CRP directly inhibits rpoS transcription (Lange and Hengge-Aronis, 1994; Kalia et al., 2013). RpoS, the stationary-phase-specific sigma factor, governs many stress responses to protect $E$. coli from some specific harmful environments including starvation, osmotic shock, and so on (Loewen et al., 1998; Battesti et al., 2011). During biofilm formation in E. coli, RpoS critically inhibits the initiation of biofilm formation, especially during the exponential growth phase (Corona-Izquierdo and Membrillo-Hernández, 2002). Thus, cAMP-CRP promotes biofilm formation by repressing $r p o S$ transcription. In conclusion, CAMP-CRP plays a major ancillary role in the regulation of biofilm formation in E. coli.

\section{CAMP-CRP REGULATES BIOFILM FORMATION IN $P$, aeruginosa}

The involvement of cAMP in the biofilm formation of $P$. aeruginosa is well-established. In contrast to $E$. coli, the preferred carbon sources of $P$. aeruginosa are acetate or tricarboxylic acid (TCA) cycle intermediates rather than the hexoses, such as glucose and fructose, that are preferred by most $\gamma$-proteobacteria (Aranda-Olmedo et al., 2005; Deutscher et al., 2006). In P. aeruginosa, carbon catabolite repression is governed by preferred carbon sources; carbon catabolite repression may be regulated by different mechanisms, such as catabolite repression control (Crc) proteins instead of cAMP-CRP (Hester et al., 2000; Aranda-Olmedo et al., 2005). A virulence factor regulator (Vfr) shares a high degree of homology to $E$. coli CRP (67\% sequence identity) and is found to be mainly involved in regulating the expression of a set of genes encoding extracellular virulence factors in P. aeruginosa (West et al., 1994; Gosset et al., 2004; Coggan and Wolfgang, 2011). Although cAMP is still the allosteric activator of Vfr, the cAMP-Vfr complex has functional 
differences from the cAMP-CRP complex in terms of response signals and regulatory mechanisms. The signals responded by cAMP-Vfr are calcium, high osmolarity, and solid surfaces rather than PTS or non-PTS sugars (Wolfgang et al., 2003; Rietsch and Mekalanos, 2006; Persat et al., 2015). In P. aeruginosa, the underlying mechanisms of cAMP-Vfr-mediated responses to solid surfaces and control biofilm formation are wellcharacterized (Figure 2). When swimming bacteria come in contact with a solid surface via their flagellum, it leads to an increase in the flagellar load, which allows these cells to become tethered to the solid surface (Schniederberend et al., 2019). This increase in flagellar load causes an interaction occurring between FlhF (a SRP-like GTPase that induces a single flagellum to assemble at the pole) and FimV (a polar organizer) (Schniederberend et al., 2019). This interaction activates CyaA and $\mathrm{CyaB}$, increasing the intracellular cAMP in the tethered bacteria (Schniederberend et al., 2019). Subsequently, cAMP activates the transcription factor Vfr and cAMP-Vfr inhibits the expression of fleQ, which encodes the master regulator of flagellar biogenesis, thereby negatively mediating the expression of flagellar genes and decreasing flagellar synthesis (Dasgupta et al., 2002; Wolfgang et al., 2003). At the same time, cAMPVfr triggers the expression of type IV pili (TFP) genes (West et al., 1994; Beatson et al., 2002; Wolfgang et al., 2003). TFP is the other mechanosensor to further sense the solid surface signal, which activates the Chp chemosensory system by the direct interaction between PilA of the TFP and PliJ of the Chp chemosensory system, which directly stimulates CyaB activity to synthesize additional cAMP (Fulcher et al., 2010; Persat et al., 2015). Finally, cAMP-Vfr further promotes the expression of TFP genes and other virulence factor genes that are involved in the biofilm formation (Wolfgang et al., 2003; Diaz et al., 2011). Therefore, the solid surface is sensed by the rotating bacterial flagellum, and this irreversible attachment requires the inhibition of flagellar synthesis coupled with increased expression of the TFP (Figure 2). In this process, cAMP-Vfr is a core regulator that mediates and integrates these pathways in $P$. aeruginosa. While numerous environmental signals can induce biofilm formation, the direct and primary signal is a solid surface (Belas, 2014). In P. aeruginosa, the flagellum acts as a mechanosensor, sensing the solid surface signal directly; this stimulates the synthesis of cAMP (Schniederberend et al., 2019). cAMP-Vfr further stimulates biofilm-inducing pathways, thereby promoting biofilm formation (Persat et al., 2015).

While CRP is the only effector of cAMP in most bacteria, a novel cAMP receptor, $\mathrm{CbpA}$, has been identified in $P$. aeruginosa (Endoh and Engel, 2009). Although the Vfr-dependent functions are not influenced by the deletion of $\operatorname{cbp} A, \mathrm{CbpA}$, which is located at the cellular poles, may be critical to the polar flagellum in a cAMP-Vfr-dependent manner (Endoh and Engel, 2009). Additionally, it has been demonstrated that glucose starvation induces $P$. aeruginosa biofilm dispersal in a cAMP-Vfr-dependent manner (Huynh et al., 2012).

In most investigations, cAMP-Vfr promotes biofilm formation in $P$. aeruginosa (Luo et al., 2015; Persat et al., 2015). However, several studies have reported conflicting results regarding cAMPVfr regulating biofilm formation in $P$. aeruginosa. Some studies have shown that a mutant with a deleted $c p d A$ gene, which encodes the CpdA protein that degrades cAMP in bacteria, had a defect in biofilm formation as compared to wild type (Ono et al., 2014; Almblad et al., 2019). Furthermore, cAMP-Vfrinhibited $P$. aeruginosa biofilm formation depended on several phosphodiesterases (PDEs), which are c-di-GMP degrading enzymes; the primary PDEs in the cell include DipA, RbdA, and BifA (Almblad et al., 2019). At the same time, it was shown that elevated levels of c-di-GMP in P. aeruginosa caused an increase in biofilm formation by reducing cAMP levels (Almblad et al., 2015). Therefore, cAMP-CRP-mediated effects on biofilm formation were produced via multiple regulation pathways in $P$. aeruginosa.

\section{CAMP-CRP REGULATES BIOFILM FORMATION IN OTHER BACTERIA}

In other members of the Enterobacteriaceae family, cAMPCRP-mediated biofilm formation differs from that in E. coli and is often complicated. For example, in S. marcescens,

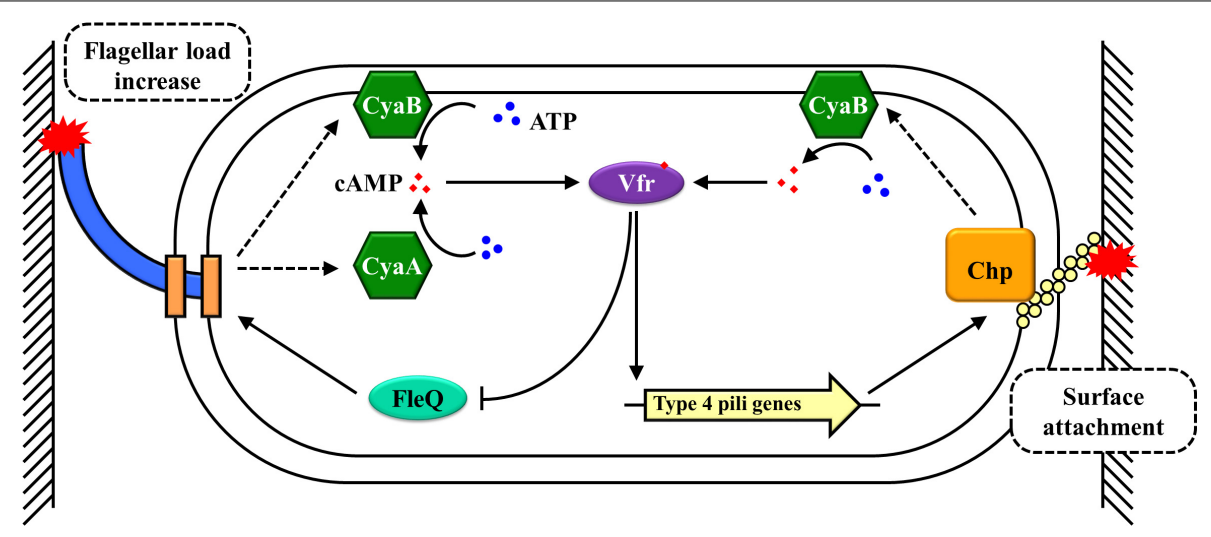

FIGURE 2 | A solid surface signal regulates biofilm formation by cAMP-CRP in P. aeruginosa. Arrowheads indicate activation, with the rear of the arrow representing inhibition. 
cAMP-CRP suppresses, instead of promoting, biofilm formation by inhibiting type 1 fimbriae synthesis (Kalivoda et al., 2008, 2013). Furthermore, although the underlying mechanisms of biofilm formation in S. enterica serovar Typhimurium are similar to those reported in E. coli, but in contrast to E. coli, cAMP-CRP downregulates the transcription of $\operatorname{csgD}$ in $S$. enterica serovar Typhimurium, which promotes pellicle formation and inhibits biofilm formation (Paytubi et al., 2017). In Klebsiella pneumoniae, cAMP-CRP promotes biofilm formation by increasing type 3 fimbriae synthesis, which is indirectly via the c-di-GMP signaling pathways (Lin et al., 2016; Ou et al., 2017; Panjaitan et al., 2019). In summary, cAMP-CRP stimulates biofilm formation in E. coli and K. pneumoniae but inhibits biofilm formation in S. marcescens. Despite this discrepancy, some of the regulatory effects of cAMP-CRP on biofilm formation are common across the Enterobacteriaceae. First, in most Enterobacteriaceae, cAMPCRP controls biofilm formation by regulating the synthesis of flagella or fimbriae, which is associated with motility (Soutourina et al., 1999; Kalivoda et al., 2008; Panjaitan et al., 2019). Second, the regulatory effects of CAMP-CRP on biofilm formation are dependent on carbon sources, due to carbon catabolite repression (Jackson et al., 2002; Sutrina et al., 2015, 2019).

In addition to the Enterobacteriaceae and P. aeruginosa, cAMP-CRP regulates biofilm formation in many other bacteria (He et al., 2007; Fong and Yildiz, 2008; Harrison et al., 2019; Ritzert et al., 2019). In Yersinia pestis, cAMP-CRP is also a central mediator of carbon catabolite repression. Although cAMP-CRP does not directly stimulate biofilm formation in this species, cAMP-CRP may indirectly promote $Y$. pestis biofilm production by facilitating the alternate carbon source expression profile, in part by regulating the carbon storage regulator protein CsrA, and stimulating another global regulator PhoP (Willias et al., 2015; Liu et al., 2016; Ritzert and Lathem, 2018; Ritzert et al., 2019). $V$. cholerae has a carbon catabolite repression system similar to that of Enterobacteriaceae, which is also regulated by cAMPCRP (Houot and Watnick, 2008). Moreover, cAMP-CRP inhibits biofilm formation in $V$. cholerae. First, cAMP-CRP activates HapR, a master regulator that suppresses biofilm formation (Fong and Yildiz, 2008; Luo et al., 2015; Sutrina et al., 2019). Second, cAMP-CRP controls biofilm formation via the c-di-GMP signaling pathways (Fong and Yildiz, 2008; Krasteva et al., 2010; Kalia et al., 2013). Biofilm maturation in $V$. cholerae requires the production of extracellular matrix components, including matrix proteins and Vibrio polysaccharide (VPS) (Casper-Lindley and Yildiz, 2004). Two vps operons are expressed as a direct result of stimulation by two positive transcriptional regulators, VpsR and VpsT, both of which are c-di-GMP effectors; the activation of these operons increases biofilm formation in V. cholerae (CasperLindley and Yildiz, 2004; Zamorano-Sánchez et al., 2015). c-diGMP does not alter the DNA-binding ability of VpsR. However, without c-di-GMP, the VpsR and RNA polymerase enzyme complex will not interact correctly with DNA and will not successfully generate an active transcription complex (Srivastava et al., 2011; Hsieh et al., 2018). VpsT is a typical c-di-GMP effector, and c-di-GMP is required for the activation of VpsT and the subsequent regulation of both $v p s$ operons (Krasteva et al., 2010). VpsR and VpsT can also activate each other, while
cAMP-CRP negatively regulates both $v p s R$ and $v p s T$ (Fong and Yildiz, 2008). Thus, cAMP-CRP controls biofilm formation via the c-di-GMP signaling pathways in $V$. cholerae.

With the exception of $P$. aeruginosa, in which cAMP-Vfr may be exclusively involve in pathogenicity, virulence, and biofilm formation, cAMP-CRP-mediated biofilm formation in most bacteria primarily depends on the cAMP-CRP-dependent pathways related to carbon metabolism. Thus, the ancillary regulation of CAMP-CRP on biofilm formation by CAMP-CRP is associated with or dependent on the carbon metabolism in many bacteria.

\section{CONCLUSION AND PERSPECTIVES}

Biofilms have emergent properties that protect their inhabitants' survival in hostile environments, such as those in which the available nutrients have changed (Myszka and Czaczyk, 2009; Zhang et al., 2014; Liu et al., 2017, 2019; Wang et al., 2017; Flemming and Wuertz, 2019). Several studies have shown that multiple bacteria form biofilms in response to changes in the types or concentrations of the available carbon sources (Myszka and Czaczyk, 2009; Zhang et al., 2014; Liu et al., 2017). The relationship between nutrient metabolism and biofilm formation is largely unknown. It is also unclear whether carbon molecules act as simple signals, stimulating biofilm formation, or whether the pathways associated with carbon metabolism are cross-regulated with those regulating biofilm formation. As a critical factor in carbon catabolite repression, cAMPCRP may be the "connecter" between the carbon metabolism and biofilm formation. This possibility should be addressed in future investigations.

Second messengers play an important role in various physiological processes in bacteria. The involvement of the second messenger c-di-GMP in biofilm formation is well established in multiple bacteria (Matsuyama et al., 2016; Liu et al., 2017), and regulatory effects of c-di-GMP on biofilm formation have been carefully reviewed (Christen et al., 2010; Dahlstrom and O'Toole, 2017; Krasteva and Sondermann, 2017). Compared to c-di-GMP, which participates in the whole biofilm cycle (from initial attachment to dispersion), the regulatory effects of cAMPCRP on biofilm formation seem mostly ancillary. However, the underlying mechanisms should also be considered. Indeed, a few studies have investigated the cross-regulation between c-di-GMP and cAMP-CRP. In K. pneumoniae, P. aeruginosa, and $V$. cholerae, cAMP-CRP/Vfr regulates the gene expression levels of PDE and DGC (c-di-GMP diguanylate cyclase) or the pathways associated with c-di-GMP (Fong and Yildiz, 2008; Lin et al., 2016; Almblad et al., 2019). However, the underlying mechanisms remain largely unknown. Future investigations should aim to characterize the pathways associated with the cross-regulation between cAMP and c-di-GMP.

\section{AUTHOR CONTRIBUTIONS}

CL, DS, JZ, and JL conducted the literature study and wrote the draft manuscript. WL edited and revised the manuscript. 


\section{FUNDING}

This study is supported by the National Natural Science Foundation of China (31900401, 31970036, and 31800020), the Natural Science Foundation of Jiangsu Province (BK20171163

\section{REFERENCES}

Almblad, H., Harrison, J. J., Rybtke, M., Groizeleau, J., Givskov, M., Parsek, M. R., et al. (2015). The cyclic AMP-Vfr signaling pathway in Pseudomonas aeruginosa is inhibited by cyclic di-GMP. J. Bacteriol. 197, 2190-2200. doi: 10.1128/JB. 00493-15

Almblad, H., Rybtke, M., Hendiani, S., Andersen, J. B., Givskov, M., and TolkerNielsen, T. (2019). High levels of cAMP inhibit Pseudomonas aeruginosa biofilm formation through reduction of the c-di-GMP content. Microbiology 165, 324-333. doi: 10.1099/mic.0.000772

Aranda-Olmedo, I., Ramos, J. L., and Marqués, S. (2005). Integration of signals through Crc and PtsN in catabolite repression of Pseudomonas putida TOL plasmid pWW0. Appl. Environ. Microbiol. 71, 4191-4198. doi: 10.1128/AEM. 71.8.4191-4198.2005

Barnhart, M. M., and Chapman, M. R. (2006). Curli biogenesis and function. Annu. Rev. Microbiol. 60, 131-147.

Battesti, A., Majdalani, N., and Gottesman, S. (2011). The RpoS-mediated general stress response in Escherichia coli. Annu. Rev. Microbiol. 65, 189-213. doi: 10.1146/annurev-micro-090110-102946

Beatson, S. A., Whitchurch, C. B., Sargent, J. L., Levesque, R. C., and Mattick, J. S. (2002). Differential regulation of twitching motility and elastase production by Vfr in Pseudomonas aeruginosa. J. Bacteriol. 184, 3605-3613. doi: 10.1128/jb. 184.13.3605-3613.2002

Belas, R. (2014). Biofilms, flagella, and mechanosensing of surfaces by bacteria. Trends Microbiol. 22, 517-527. doi: 10.1016/j.tim.2014. 05.002

Brombacher, E., Dorel, C., Zehnder, A. J. B., and Landini, P. (2003). The curli biosynthesis regulator CsgD co-ordinates the expression of both positive and negative determinants for biofilm formation in Escherichia coli. Microbiology 149, 2847-2857. doi: 10.1099/mic.0.26306-0

Casper-Lindley, C., and Yildiz, F. H. (2004). VpsT Is a transcriptional regulator required for expression of $v p s$ biosynthesis genes and the development of rugose colonial morphology in Vibrio cholerae O1 E1 Tor. J. Bacteriol. 186, 1574-1578. doi: 10.1128/jb.186.5.1574-1578.2004

Chevance, F. F., and Hughes, K. T. (2008). Coordinating assembly of a bacterial macromolecular machine. Nat. Rev. Microbiol. 6, 455-465. doi: 10.1038/ nrmicro1887

Christen, M., Kulasekara, H. D., Christen, B., Kulasekara, B. R., Hoffman, L. R., and Miller, S. I. (2010). Asymmetrical distribution of the second messenger c-diGMP upon bacterial cell division. Science 328, 1295-1297. doi: 10.1126/science. 1188658

Coggan, K. A., and Wolfgang, M. C. (2011). Global regulatory pathways and crosstalk control Pseudomonas aeruginosa environmental lifestyle and virulence phenotype. Curr. Issues Mol. Biol. 14, 47-70.

Corona-Izquierdo, F. P., and Membrillo-Hernández, J. (2002). A mutation in rpoS enhances biofilm formation in Escherichia coli during exponential phase of growth. FEMS Microbiol. Lett. 211, 105-110. doi: 10.1111/j.1574-6968.2002. tb11210.x

Dahlstrom, K. M., and O’Toole, G. A. (2017). A symphony of cyclases: specificity in diguanylate cyclase signaling. Annu. Rev. Microbiol. 71, 179-195. doi: 10.1146/ annurev-micro-090816-093325

Dasgupta, N., Ferrell, E. P., Kanack, K. J., West, S. E., and Ramphal, R. (2002).fleQ, the gene encoding the major flagellar regulator of Pseudomonas aeruginosa, is $\sigma 70$ dependent and is downregulated by Vfr, a homolog of Escherichia coli cyclic AMP receptor protein. J. Bacteriol. 184, 5240-5250. doi: 10.1128/jb.184. 19.5240-5250.2002

Deutscher, J., Francke, C., and Postma, P. W. (2006). How phosphotransferase system-related protein phosphorylation regulates carbohydrate metabolism in bacteria. Microbiol. Mol. Biol. Rev. 70, 939-1031. doi: 10.1128/MMBR. 00024-06 and BK20181009), the Natural Science Foundation of Xuzhou city (KC19196), the Six Talent Peaks Project of Jiangsu Province (JNHB-103), the Qing Lan Project of Jiangsu Province, Priority Academic Program Development of Jiangsu Higher Education Institutions.

Diaz, M. R., King, J. M., and Yahr, T. L. (2011). Intrinsic and extrinsic regulation of type III secretion gene expression in Pseudomonas aeruginosa. Front. Microbiol. 2:89. doi: $10.3389 /$ fmicb.2011.00089

Endoh, T., and Engel, J. N. (2009). CbpA: a polarly localized novel cyclic AMPbinding protein in Pseudomonas aeruginosa. J. Bacteriol. 191, 7193-7205. doi: 10.1128/JB.00970-09

Flemming, H. C., Wingender, J., Szewzyk, U., Steinberg, P., Rice, S. A., and Kjelleberg, S. (2016). Biofilms: an emergent form of bacterial life. Nat. Rev. Microbiol. 14, 563-575. doi: 10.1038/nrmicro.2016.94

Flemming, H.-C., and Wuertz, S. (2019). Bacteria and archaea on Earth and their abundance in biofilms. Nat. Rev. Microbiol. 17, 247-260. doi: 10.1038/s41579019-0158-9

Fong, J. C., and Yildiz, F. H. (2008). Interplay between cyclic AMP-cyclic AMP receptor protein and cyclic di-GMP signaling in Vibrio cholerae biofilm formation. J. Bacteriol. 190, 6646-6659. doi: 10.1128/JB.00466-08

Fulcher, N. B., Holliday, P. M., Klem, E., Cann, M. J., and Wolfgang, M. C. (2010). The Pseudomonas aeruginosa Chp chemosensory system regulates intracellular cAMP levels by modulating adenylate cyclase activity. Mol. Microbiol. 76, 889-904. doi: 10.1111/j.1365-2958.2010.07135.x

Gosset, G., Zhang, Z., Nayyar, S., Cuevas, W. A., and Saier, M. H. (2004). Transcriptome analysis of Crp-dependent catabolite control of gene expression in Escherichia coli. J. Bacteriol. 186, 3516-3524. doi: 10.1128/JB.186.11.35163524.2004

Guttenplan, S. B., and Kearns, D. B. (2013). Regulation of flagellar motility during biofilm formation. FEMS Microbiol. Rev. 37, 849-871. doi: 10.1111/1574-6976. 12018

Ha, D. G., and O’Toole, G. A. (2015). c-di-GMP and its effects on biofilm formation and dispersion: a Pseudomonas aeruginosa review. Microbiol. Spectr. 3:MB-0003-2014. doi: 10.1128/microbiolspec.MB-0003-2014

Harrison, A., Hardison, R. L., Wallace, R. M., Fitch, J., Heimlich, D. R., Bryan, M. O., et al. (2019). Reprioritization of biofilm metabolism is associated with nutrient adaptation and long-term survival of Haemophilus influenzae. NPJ Biofilms Microbiomes 5:33. doi: 10.1038/s41522-019-0105-6

He, Y. W., Ng, A. Y., Xu, M., Lin, K., Wang, L. H., Dong, Y. H., et al. (2007). Xanthomonas campestris cell-cell communication involves a putative nucleotide receptor protein $\mathrm{Clp}$ and a hierarchical signalling network. Mol. Microbiol. 64, 281-292. doi: 10.1111/j.1365-2958.2007.05670.x

Hester, K. L., Lehman, J., Najar, F., Song, L., Roe, B. A., Macgregor, C. H., et al. (2000). Crc is involved in catabolite repression control of the bkd operons of Pseudomonas putida and Pseudomonas aeruginosa. J. Bacteriol. 182, 1144-1149. doi: 10.1128/jb.182.4.1144-1149.2000

Hogema, B. M., Arents, J. C., Inada, T., Aiba, H., and Postma, P. W. (1997). Catabolite repression by glucose 6-phosphate, gluconate and lactose in Escherichia coli. Mol. Microbiol. 24, 857-867. doi: 10.1046/j.1365-2958.1997. 3991761.x

Houot, L., and Watnick, P. I. (2008). A novel role for enzyme I of the Vibrio cholerae phosphoenolpyruvate phosphotransferase system in regulation of growth in a biofilm. J. Bacteriol. 190, 311-320. doi: 10.1128/JB.01410-07

Hsieh, M. L., Hinton, D. M., and Waters, C. M. (2018). VpsR and cyclic diGMP together drive transcription initiation to activate biofilm formation in Vibrio cholerae. Nucleic Acids Res. 46, 8876-8887. doi: 10.1093/nar/ gky606

Hufnagel, D. A., Evans, M. L., Greene, S. E., Pinkner, J. S., Hultgren, S. J., and Chapman, M. R. (2016). The catabolite repressor protein-cyclic AMP complex regulates $c s g D$ and biofilm formation in uropathogenic Escherichia coli. J. Bacteriol. 198, 3329-3334. doi: 10.1128/JB.00652-16

Huynh, T. T., McDougald, D., Klebensberger, J., Al Qarni, B., Barraud, N., Rice, S. A., et al. (2012). Glucose starvation-induced dispersal of Pseudomonas aeruginosa biofilms is cAMP and energy dependent. PLoS One 7:e42874. doi: 10.1371/journal.pone.0042874 
Jackson, D. W., Simecka, J. W., and Romeo, T. (2002). Catabolite repression of Escherichia coli biofilm formation. J. Bacteriol. 184, 3406-3410. doi: 10.1128/jb. 184.12.3406-3410.2002

Jenal, U., Reinders, A., and Lori, C. (2017). Cyclic di GMP: second messenger extraordinaire. Nat. Rev. Microbiol. 15, 271-284. doi: 10.1038/nrmicro. 2016.190

Kalia, D., Merey, G., Nakayama, S., Zheng, Y., Zhou, J., Luo, Y., et al. (2013). Nucleotide, c-di-GMP, c-di-AMP, cGMP, cAMP, (p)ppGpp signaling in bacteria and implications in pathogenesis. Chem. Soc. Rev. 42, 305-341. doi: 10.1039/ c2cs35206k

Kalivoda, E. J., Brothers, K. M., Stella, N. A., Schmitt, M. J., and Shanks, R. M. (2013). Bacterial cyclic AMP-phosphodiesterase activity coordinates biofilm formation. PLoS One 8:e71267. doi: 10.1371/journal.pone.0071267

Kalivoda, E. J., Stella, N. A., O’Dee, D. M., Nau, G. J., and Shanks, R. M. (2008). The cyclic AMP-dependent catabolite repression system of Serratia marcescens mediates biofilm formation through regulation of type 1 fimbriae. Appl. Environ. Microbiol. 74, 3461-3470. doi: 10.1128/AEM.02733-07

Krasteva, P. V., Fong, J. C., Shikuma, N. J., Beyhan, S., Navarro, M. V., Yildiz, F. H., et al. (2010). Vibrio cholerae VpsT regulates matrix production and motility by directly sensing cyclic di-GMP. Science 327, 866-868. doi: 10.1126/science. 1181185

Krasteva, P. V., and Sondermann, H. (2017). Versatile modes of cellular regulation via cyclic dinucleotides. Nat. Chem. Biol. 13, 350-359. doi: 10.1038/nchembio. 2337

Lange, R., and Hengge-Aronis, R. (1994). The cellular concentration of the sigma $\mathrm{S}$ subunit of RNA polymerase in Escherichia coli is controlled at the levels of transcription, translation, and protein stability. Genes Dev. 8, 1600-1612. doi: $10.1101 / \mathrm{gad} .8 .13 .1600$

Lin, C. T., Lin, T. H., Wu, C. C., Wan, L., Huang, C. F., and Peng, H. L. (2016). CRP-cyclic AMP regulates the expression of type 3 fimbriae via cyclic di-GMP in Klebsiella pneumoniae. PLoS One 11:e0162884. doi: 10.1371/journal.pone. 0162884

Liu, C., Sun, D., Zhu, J., and Liu, W. (2019). Two-component signal transduction systems: a major strategy for connecting input stimuli to biofilm formation. Front. Microbiol. 9:3279. doi: 10.3389/fmicb.2018.03279

Liu, C., Yang, J., Liu, L., Li, B., Yuan, H., and Liu, W. (2017). Sodium lactate negatively regulates Shewanella putrefaciens CN32 biofilm formation via a three-component regulatory System (LrbS-LrbA-LrbR). Appl. Environ. Microbiol. 83:e00712-17. doi: 10.1128/AEM.00712-17

Liu, L., Fang, H., Yang, H., Zhang, Y., Han, Y., Zhou, D., et al. (2016). CRP is an activator of Yersinia pestis biofilm formation that operates via a mechanism involving gmhA and waaAE-coaD. Front. Microbiol. 7:295. doi: 10.3389/fmicb. 2016.00295

Loewen, P. C., Hu, B., Strutinsky, J., and Sparling, R. (1998). Regulation in the rpoS regulon of Escherichia coli. Can. J. Microbiol. 44, 707-717.

Luo, Y., Zhao, K., Baker, A. E., Kuchma, S. L., Coggan, K. A., Wolfgang, M. C., et al. (2015). A hierarchical cascade of second messengers regulates Pseudomonas aeruginosa surface behaviors. mBio 6:e2456-14. doi: 10.1128/mBio.02456-14

Matsuyama, B. Y., Krasteva, P. V., Baraquet, C., Harwood, C. S., Sondermann, H., and Navarro, M. V. (2016). Mechanistic insights into c-di-GMP-dependent control of the biofilm regulator FleQ from Pseudomonas aeruginosa. Proc. Natl. Acad. Sci. U.S.A. 113, E209-E218. doi: 10.1073/pnas.1523148113

Müller, C. M., Aberg, A., Straseviçiene, J., Emody, L., Uhlin, B. E., and Balsalobre, C. (2009). Type 1 fimbriae, a colonization factor of uropathogenic Escherichia coli, are controlled by the metabolic sensor CRP-cAMP. PLoS Pathog. 5:e1000303. doi: 10.1371 /journal.ppat. 1000303

Myszka, K., and Czaczyk, K. (2009). Characterization of adhesive exopolysaccharide (EPS) produced by Pseudomonas aeruginosa under starvation conditions. Curr. Microbiol. 58, 541-546. doi: 10.1007/s00284-0099365-3

Ogasawara, H., Yamamoto, K., and Ishihama, A. (2011). Role of the biofilm master regulator CsgD in cross-regulation between biofilm formation and flagellar synthesis. J. Bacteriol. 193, 2587-2597. doi: 10.1128/JB.01468-10

Ono, K., Oka, R., Toyofuku, M., Sakaguchi, A., Hamada, M., Yoshida, S., et al. (2014). cAMP signaling affects irreversible attachment during biofilm formation by Pseudomonas aeruginosa PAO1. Microbes Environ. 29, 104-106. doi: 10.1264/jsme2.me13151

Ou, Q., Fan, J., Duan, D., Xu, L., Wang, J., Zhou, D., et al. (2017). Involvement of cAMP receptor protein in biofilm formation, fimbria production, capsular polysaccharide biosynthesis and lethality in mouse of Klebsiella pneumoniae serotype K1 causing pyogenic liver abscess. J. Med. Microbiol. 66, 1-7. doi: 10.1099/jmm.0.000391

Panjaitan, N. S. D., Horng, Y. T., Cheng, S. W., Chung, W. T., and Soo, P. C. (2019). EtcABC, a putative EII complex, regulates type 3 fimbriae via CRPcAMP signaling in Klebsiella pneumoniae. Front. Microbiol. 10:1558. doi: 10. 3389/fmicb.2019.01558

Park, Y. H., Lee, B. R., Seok, Y. J., and Peterkofsky, A. (2006). In vitro reconstitution of catabolite repression in Escherichia coli. J. Biol. Chem. 281, 6448-6454. doi: 10.1074/jbc.M512672200

Paytubi, S., Cansado, C., Madrid, C., and Balsalobre, C. (2017). Nutrient composition promotes switching between pellicle and bottom biofilm in Salmonella. Front. Microbiol. 8:2160. doi: 10.3389/fmicb.2017.02160

Persat, A., Inclan, Y. F., Engel, J. N., Stone, H. A., and Gitai, Z. (2015). Type IV pili mechanochemically regulate virulence factors in Pseudomonas aeruginosa. Proc. Natl. Acad. Sci. U.S.A. 112, 7563-7568. doi: 10.1073/pnas.1502025112

Purcell, E. B., Tamayo, R., and Shen, A. (2016). Cyclic diguanylate signaling in Gram-positive bacteria. FEMS Microbiol. Rev. 40, 753-773. doi: 10.1093/femsre/ fuw013

Rietsch, A., and Mekalanos, J. J. (2006). Metabolic regulation of type III secretion gene expression in Pseudomonas aeruginosa. Mol. Microbiol. 59, 807-820. doi: 10.1111/j.1365-2958.2005.04990.x

Ritzert, J. T., and Lathem, W. W. (2018). Depletion of glucose activates catabolite repression during pneumonic plague. J. Bacteriol. 200:e00737-17. doi: 10.1128/ JB.00737-17

Ritzert, J. T., Minasov, G., Embry, R., Schipma, M. J., and Satchell, K. J. F. (2019). The cyclic AMP receptor protein regulates quorum sensing and global gene expression in Yersinia pestis during planktonic growth and growth in biofilms. mBio 10:e02613-19. doi: 10.1128/mBio.02613-19

Rudd, K. E. (2000). EcoGene: a genome sequence database for Escherichia coli K-12. Nucleic Acids Res. 28, 60-64. doi: 10.1093/nar/28.1.60

Schniederberend, M., Williams, J. F., Shine, E., Shen, C., Jain, R., Emonet, T., et al. (2019). Modulation of flagellar rotation in surface-attached bacteria: a pathway for rapid surface-sensing after flagellar attachment. PLoS Pathog. 15:e1008149. doi: 10.1371/journal.ppat.1008149

Soutourina, O., Kolb, A., Krin, E., Laurentwinter, C., Rimsky, S., Danchin, A., et al. (1999). Multiple control of flagellum biosynthesis in Escherichia coli: role of H-NS protein and the cyclic AMP-catabolite activator protein complex in transcription of the flhDC master operon. J. Bacteriol. 181, 7500-7508.

Srivastava, D., Harris, R. C., and Waters, C. M. (2011). Integration of cyclic diGMP and quorum sensing in the control of vpsT and aphA in Vibrio cholerae. J. Bacteriol. 193, 6331-6341. doi: 10.1128/JB.05167-11

Sutrina, S. L., Callender, S., Grazette, T., Scantlebury, P., O’neal, S., Thomas, K., et al. (2019). The quantity and distribution of biofilm growth of Escherichia coli strain ATCC 9723 depends on the carbon/energy source. Microbiology 165, 47-64. doi: 10.1099/mic.0.000745

Sutrina, S. L., Daniel, K., Lewis, M., Charles, N. T., Anselm, C. K., Thomas, N., et al. (2015). Biofilm growth of Escherichia coli is subject to cAMP-dependent and cAMP-independent inhibition. J. Mol. Microbiol. Biotechnol. 25, 209-225. doi: $10.1159 / 000375498$

Valentini, M., and Filloux, A. (2016). Biofilms and cyclic di-GMP (c-di-GMP) signaling: lessons from Pseudomonas aeruginosa and other bacteria. J. Biol. Chem. 291, 12547-12555. doi: 10.1074/jbc.R115.711507

Wang, D., Xu, A., Elmerich, C., and Ma, L. Z. (2017). Biofilm formation enables free-living nitrogen-fixing rhizobacteria to fix nitrogen under aerobic conditions. ISME J. 11, 1602-1613. doi: 10.1038/ismej. 2017.30

West, S. E., Sample, A. K., and Runyen-Janecky, L. J. (1994). The vfr gene product, required for Pseudomonas aeruginosa exotoxin A and protease production, belongs to the cyclic AMP receptor protein family. J. Bacteriol. 176, 7532-7542. doi: 10.1128/jb.176.24.7532-7542.1994

Willias, S. P., Chauhan, S., Lo, C. C., Chain, P. S., and Motin, V. L. (2015). CRPmediated carbon catabolite regulation of Yersinia pestis biofilm formation is enhanced by the carbon storage regulator protein, CsrA. PLoS One 10:e135481. doi: 10.1371/journal.pone.0135481

Wolfgang, M. C., Lee, V. T., Gilmore, M. E., and Lory, S. (2003). Coordinate regulation of bacterial virulence genes by a novel adenylate cyclase-dependent signaling pathway. Dev. Cell 4, 253-263. doi: 10.1016/s1534-5807(03) 00019-4 
Xie, L., Altindal, T., Chattopadhyay, S., and Wu, X. L. (2011). From the Cover: bacterial flagellum as a propeller and as a rudder for efficient chemotaxis. Proc. Natl. Acad. Sci. U.S.A. 108, 2246-2251. doi: 10.1073/pnas.1011953108

Zamorano-Sánchez, D., Fong, J. C., Kilic, S., Erill, I., and Yildiz, F. H. (2015). Identification and characterization of VpsR and VpsT binding sites in Vibrio cholerae. J. Bacteriol. 197, 1221-1235. doi: 10.1128/JB.02439-14

Zhang, W., Seminara, A., Suaris, M., Brenner, M. P., Weitz, D. A., and Angelini, T. E. (2014). Nutrient depletion in Bacillus subtilis biofilms triggers matrix production. New J. Phys. 16, 83-97. doi: 10.1088/1367-2630/16/1/ 015028
Conflict of Interest: The authors declare that the research was conducted in the absence of any commercial or financial relationships that could be construed as a potential conflict of interest.

Copyright (c) $2020 \mathrm{Liu}, \mathrm{Sun}, \mathrm{Zhu}$, Liu and Liu. This is an open-access article distributed under the terms of the Creative Commons Attribution License (CC BY). The use, distribution or reproduction in other forums is permitted, provided the original author(s) and the copyright owner(s) are credited and that the original publication in this journal is cited, in accordance with accepted academic practice. No use, distribution or reproduction is permitted which does not comply with these terms. 